\section{Controversies concerning mitochondrial replacement therapy}

Research on mitochondrial replacement therapy (MRT) holds the promise of helping women who have, or are at risk of transmitting, mitochondrial disease, but has recently been blocked by the Food and Drug Administration (FDA). Thus, several critical ethical and policy questions arise. Mitochondrial disease can be devastating for those who have it. Yet existing treatments remain supportive rather than curative. Women confronting this disease have a high chance of having an affected child and limited reproductive alternatives.

Recently, US and British researchers have been developing MRT in animal models as a way of possibly preventing inheritance of mitochondrial mutations. MRT offers women with mitochondrial disease the chance to have a genetically related child without mitochondrial disease by placing the nuclear DNA from the mother's egg into a donor egg that has no identified mitochondrial mutations. Researchers are investigating two MRT techniques: pronuclear transfer and maternal spindle transfer, both of which, if successful, would result in an embryo whose nucleus is formed by the normal mixture of two parental gamete nuclei but whose mitochondrial genome is from a third individual.

Many scientists and patient advocacy groups have thus called for MRT trials in humans. The UK government has published draft regulations for consultation that would allow clinical trials of MRT (1). Such trials would presumably be observational studies of outcomes after intervention. The number of participants would be limited until initial data are analyzed on those with the highest risk of transmitting severe mitochondrial disease. In the United Kingdom, only 10 applicants are predicted to be eligible per year (1). Data could subsequently be gathered and analyzed through birth onward to demonstrate that mitochondrial mutations or other major abnormalities are not transmitted. These offspring would then be followed over time. Ideally, their eventual offspring would be studied as well. Pending the results of the initial phases of this study (e.g., observations of infants born using the procedure), MRT could potentially be performed and studied with additional participants with appropriate careful follow-up as well (i.e., 40 or more years of longitudinal multigenerational study would not necessarily need to be completed before performing and studying MRT on additional participants).

In the United States, the FDA met in February 2014 and collected comments through May 2014 and has suggested that it will continue to ban human trials. Here we discuss the arguments offered against studies of MRT and provide reasons why clinical studies are ethically justified.

\section{OBJECTIONS TO MRT}

\section{Possible Biological Risks}

MRT opponents have raised several concerns, including possible harms from the disruption of specific mitochondrial and nuclear genome interactions, based on possible evidence from heavily in-bred laboratory mice and fruit flies (2). Other researchers have countered these arguments, pointing out that macaque studies suggest such harmful interactions are "unlikely" (3), the relevance of the laboratory data to humans also remains unclear, and compensatory mutations may exist in humans (4). No evidence has been found of such interactions causing any disease in humans (3). However, longitudinal follow-up data have not been published on humans created using three parents. In the late 1990s, approximately 30 babies in the United States and elsewhere were born after ooplasm transfer-with ooplasm from donor oocytes injected into the oocytes of women with repeated IVF failure. However in 2001, the FDA stated that it would have to approve any further use of this practice, which thus ceased (5). Mitochondrial haplotypes between mitochondrial donor and the mother can also be matched in clinical trials to avoid any such problems (3), and potential patients can be informed of these and other potential risks. In response to these concerns, in June 2014, UK policy makers requested more information about recent scientific advances concerning haplotypes but reiterated that nothing indicates that MRT is unsafe (1) and suggested that mitochondrial DNA haplogroup matching might also be considered. Another potential fear is that, if allowed, MRT may in the future be expanded into other areas such as age-related infertility or nongenetic disorders. However, in the United Kingdom, the current draft regulations explicitly state that the only indication is mitochondrial disease, creating a clear legal barrier to such fears being realized. In addition, the fact that ooplasm transfer ceased after the FDA's decision about it (as described earlier) suggests that concerns regarding potential misuses of MRT for unlicensed indications are unsupported by past experience.

MRT opponents also highlight potential harms to egg donors. Yet, after 30 years of refinement, possible harms to donors are generally well characterized, relatively low, and well described in informed consent documents. Currently, many women regularly donate eggs voluntarily for reproductive purposes. It would, arguably, be unnecessarily restrictive and paternalistic to prevent women from donating eggs if they wish to help other women have children free of mitochondrial disease.

In general, questions of how much evidence is needed before any new technology is investigated in humans are often controversial. What constitutes acceptable levels of possible risk versus benefit before human trials start varies widely in different areas of research. Human trials frequently proceed in the face of considerable uncertainty. Risks are minimized but not always eliminated and are included in informed consent to allow prospective subjects to make fully informed decisions. MRT proponents thus feel that the current needs outweigh the risks and that the autonomy of women who without MRT face risks of having children with severe mitochondrial disease should be respected, by permitting them to make these informed decisions for themselves.

Opponents also fear legal problems related to perceptions that offspring would have "three parents." Yet most legal 
scholars disagree, drawing on evidence and theory from gamete donation and surrogacy. Moreover, the recent draft regulations in the United Kingdom explicitly state that the mitochondrial donor would be considered akin to an organ donor and not legally a parent (1).

MRT critics claim, too, that the child might suffer owing to knowledge of his or her genetic origins. However, as in the case with donated eggs or sperm, which involve far more nonparental genetic contribution, children would ideally be informed about the donation. Such openness has not been found to harm these offspring (6).

Opponents also claim that embryos and adults have equal moral status and that conducting research on any embryo cannot be ethically justified, thereby ruling out MRT. But MRT proponents argue that the embryo has increasing moral status as it develops and that before a certain stage (e.g., 14 days postfertilization) research on embryos to alleviate human suffering is ethically permissible. Moreover, MRT requires unfertilized oocytes, not embryos, and as such does not involve researchers altering existing embryos-thus avoiding questions concerning the moral status of the embryo.

\section{Legal and Ethical Issues Concerning Modification of the Germ Line}

Critics claim that MRT "crosses the Rubicon" of germ line modification, intentionally manipulating the germ line, and is thus contrary to human dignity, a slippery slope toward impermissible genetic enhancement. These concerns often reflect fears of physicians "playing God" and claims that offspring have a "right to an unmanipulated genome." These arguments frequently seek to invoke science fiction-type scenarios to try to instill fear (e.g., of genetic elites using genetic enhancements). Yet these scenarios will by no means necessarily occur. In the past, physicians have been accused of "playing God" when using new technologies with limited availability (e.g., renal dialysis machines). The development of appropriate guidelines and standards abated these concerns. Moreover, preventing transmission of identified lethal genetic mutations clearly differs from potential so-called genetic enhancement based on nonmedical traits. Partly because the mitochondrial genome is physically separate from the nuclear genome and much smaller, clear workable regulations based on these distinctions are possible and should not a priori impede all potentially beneficial research. Additionally, to prioritize a "right" to an unaltered genome or to accept the slippery slope argument condemns people to be born with serious, life-shortening diseases that could be preventable.

\section{Stigmatization}

Critics argue additionally that MRT will stigmatize current mitochondrial disorders patients. Yet such an argument could be applied to any research on interventions that may aid future generations of patients.

\section{Use of Resources}

Opponents assert, too, that since the number of families likely to benefit is relatively small, resources could be better used elsewhere. However, MRT would have large net benefits to society due to the reduction in costs associated with having mitochondria disease and direct financial benefits to the parents and the health care system (1). Moreover, families may use their own resources to pursue MRT (as with preimplantation genetic diagnosis), minimizing public expenditures while increasing scientific knowledge and experience.

Critics insist, as well, that prospective parents could instead adopt children. But many such individuals face logistical and bureaucratic barriers to doing so and prefer genetically related children. Although MRT opponents feel that this preference does not justify the imposition of risks on future children, such future risks remain conjectural, and parents might otherwise have children who would suffer from disease. Moreover, desires for a genetic link, alleviation of serious disease, and respect for potential patients' autonomy arguably outweigh paternalistic concerns about these possible risks.

\section{ARGUMENTS IN FAVOR OF MRT Prevention of Disease}

Importantly, MRT uniquely provides women with mitochondrial mutations the ability to have genetically related children free from this devastating disease. Prevention of severe disease among children genetically linked to their parents can further enhance both individual and societal well-being. Indeed, failure to fully consider trials in humans is arguably unjust.

\section{Physicians' Duties}

Physicians have a duty to act to benefit their patients-both prospective parents and children. Allowing patients to fulfill desires for genetically related children and reducing the incidence of significant disease are consistent with this duty. The risks of MRT do not appear to be so great as to prohibit initial research involving humans, as described above; and to deny women with mitochondrial disease the same opportunity to have a genetically related child as other women would be unjust.

\section{CONCLUSIONS}

Alhough arguments against MRT research have been proposed, and appear to be shaping the FDA's approach, we would argue that the potential benefits appear to outweigh the potential risks, and that respect for these women's autonomy and physicians' duties toward beneficence further support this research. Specifically, the potential risks of carefully designed and monitored clinical studies of MRT in humans remain speculative, can be minimized or prevented, and are outweighed by the potential benefits: reducing mitochondrial disease and its devastating effects in children, increasing reproductive options for women confronting this disease, and fulfilling doctors' duties toward 
their patients. Appropriate informed consent would also be obtained. Although women with mitochondrial disease could potentially adopt children instead, the desire for genetically related children deserves respect, and arguments to refuse these prospective parents assistance deny these individuals' autonomy and impede the fundamental principles of beneficence and justice. Appropriate regulation, ongoing research on risks among humans and other species, and commitment to abolishing stigma and discrimination relating to mitochondrial and other diseases should also be pursued. Such research on MRT thus appears reasonable and deserves support.

Robert Klitzman, M.D. ${ }^{a}$ Mark Toynbee, M.B.B.S. ${ }^{b}$ Mark V. Sauer, M.D. ${ }^{c}$

${ }^{a}$ Masters of Bioethics Program, Columbia University, New York, New York;

b Department of Psychiatry, University of 0xford, Oxford, United Kingdom; and

c Department of Obstetrics and Gynecology and Center for Women's Reproductive Care, Columbia University, New York, New York

http://dx.doi.org/10.1016/j.fertnstert.2014.10.028

You can discuss this article with its authors and with other ASRM members at http://fertstertforum.com/klitzmanr-controversiesmitochondrial-replacement-therapy/

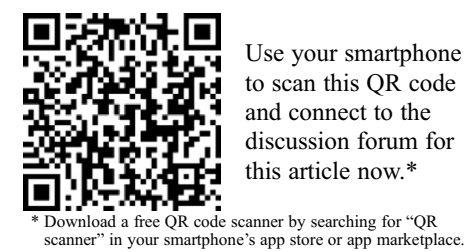

\section{REFERENCES}

1. Department of Health, UK. Mitochondrial donation: a consultation on draft regulations to permit the use of new treatment techniques to prevent the transmission of a serious mitochondrial disease from mother to child, February 2014. Available at: https://www.gov.uk/government/uploads/system/uploads/ attachment_data/file/285251/mitochondrial_donation_consultation_document_ 240214 Accessible V0.4.pdf. Accessed September 24, 2014.

2. Reinhardt K, Dowling DK, Morrow EH. Policy forum: mitochondrial replacement, evolution, and the clinic. Science 2013;341:1345-6.

3. Chinnery PF, Craven L, Mitalipov S, Stewart JB, Herbert M, Turnbull DM. The challenges of mitochondrial replacement. PLoS Genet 2014;10:e1004315.

4. Beekman M, Dowling DK, Aanen DK. The costs of being male: are there sex-specific effects of uniparental mitochondrial inheritance? Philos Trans $R$ Soc Lond B Biol Sci 2014;369:20130440.

5. Zoon KC. Letter to sponsors/researchers: Human cells used in therapy involving the transfer of genetic material by means other than the union of gamete nuclei. Available at: http://www.fda.gov/BiologicsBloodVaccines/ SafetyAvailability/ucm105852.htm. Accessed September 24, 2014.

6. Golombok S, Readings J, Blake L, Casey P, Mellish L, Marks A, et al. Children conceived by gamete donation: psychological adjustment and mother-child relationships at age 7. J Fam Psychol 2011;25:230-9. 